Case Reports

\title{
Altered Passive Eruption: A Combined Perio-Restorative Approach: A Case Report
}

\author{
${ }^{1}$ Farah Antar, ${ }^{1}$ Racha Hajj, ${ }^{2}$ Maroun Dagher and ${ }^{1}$ Elie Zebouni \\ ${ }^{I}$ Department of Prosthodontics and Restorative Dentistry, Saint Joseph University, Beirut- Lebanon \\ ${ }^{2}$ Department of Periodontology, Saint Joseph University, Beirut-Lebanon
}

Article history

Received: 27-01-2021

Revised: 01-04-2021

Accepted: 05-04-2021

Corresponding Author:

Farah Antar

Department of Prosthodontics

and Restorative Dentistry, Saint

Joseph University, Beirut-

Lebanon

Email: drfarahantar@hotmail.com

\begin{abstract}
Altered passive eruption is described as a condition in which the relationship between teeth, alveolar bone and the soft tissues creates an excessive gingival display and, in turn, in some circumstances, it may reveal a clinical aspect known as the gummy smile. A 29 -year-old male patient presented to a dental clinic in Beirut, Lebanon, complaining about short looking teeth and excessive gingival display while smiling. Before choosing the adequate treatment and starting our procedure, periodontal and esthetic factors were analyzed. The patient was diagnosed with altered passive eruption. The treatment plan consisted of 2 phases: Surgical phase: Crown lengthening prosthetic phase: Lithium discilicate veneers the aim of this paper is to describe the management of a case diagnosed with altered passive eruption. Altered passive eruption, one of the conditions that can lead to gummy smile is defined as a dent gingival relationship in which the gingival margin is positioned coronally to the cement enamel junction. A well performed clinical exam, leading to a correct diagnosis and therefore an adequate treatment plan will enhance the post-operative results.
\end{abstract}

Keywords: Altered Passive Eruption, Alveolar Crest, Cemento Enamel Junction, Crown Lengthening, Gingival Zenith, Mucoperiostal Flap, Veneers

\section{Introduction}

Achieving an esthetic smile with gingival harmony depends on many factors such as the lip form, the smile symmetry, the teeth dimension, the gingival zenith and the gingival display (Madaan et al., 2015). The relationship between teeth and gingival tissues has been emphasized by (Garber and Salama, 1996) as a major component of a beautiful smile. An esthetic smile requires a harmony between the gingival levels of the teeth; for central incisors and canines, the gingival zenith lies at the same level/height whereas for lateral incisors it is slightly coronal to the central incisor. According to (Allen, 1988) 2 to $3 \mathrm{~mm}$ of gingival exposure was perceived as an esthetically pleasing smile by patients, whereas an excessive exposure $(>3 \mathrm{~mm})$ was perceived as unesthetic. An excessive exposure is called gummy smile and can be the result of many conditions: Vertical maxillary excess, hypotonic short upper lip, hyperfunctional upper lip and insufficient clinical crown height (Madaan et al., 2015). One of the causes of insufficient crown height is the Altered Passive Eruption (APE), also called retarded passive eruption, delayed passive eruption or incomplete passive eruption. Many etiological factors are attributed to this condition:
Deficiency of growth hormone, epidermal growth factor, insulin-like growth factors I and II and hypopituitarism causing delayed dental eruption (Alpiste-Illueca, 2011).

APE was first described by (Coslet et al., 1977) as the clinical condition where the gingival margin does not migrate to its final position on the cemental surface but remains positioned on, or near the enamel surface and it was classified in two types based on the gingival/anatomic crown relationship and two subtypes based on the cementoenamel junction/alveolar crest relationship (Coslet et al., 1977). In type 1 , there is a noticeably wider band of keratinized tissue than type 2 and in the A subgroup, the osseous crest is 1.5 to $2 \mathrm{~mm}$ apical to the CEJ, while in the Subgroup B, the osseous crest is directly adjacent to the CEJ. The aim of this article is to describe the sequential multidisciplinary approach to the treatment of an type 1, subgroup B case of altered passive eruption.

\section{Case Report}

A 29-year-old young adult male patient presented to the dental clinic complaining about short looking teeth and excessive gingival display while smiling. The medical history revealed a healthy patient, while the dental history showed that the patient had a number of root canal therapies, 2 implants and crowns more than 2 years ago. 
The patient was seen for follow up and maintenance appointments by his former treating dentist. His last appointment was 2 months ago when he was referred to our dental office for further treatment (Figs. 1 and 2)

\section{Clinical Examination}

Clinically, his extraoral examination showed that he had a facial symmetry, a medial smile line (lip line position) and normal lip thickness and tonicity. Intraorally, the patient had a marginal gingivitis (Full mouth plaque score $<20 \%$ and full mouth bleeding index $<20 \%$ ), with short teeth in relation to the gingival margin and an excess of keratinized gingiva.

\section{Radiographic Examination}

The panoramic x-ray revealed less than $0,5-1 \mathrm{~mm}$ distance between the CEJ and the alveolar crest radiographic ally, with no bone loss (Fig. 3).

\section{Diagnosis and Treatment Plan}

Based on the clinical examination and the available radiograph, a treatment plan for the diagnosed APE type 1 , subgroup B was adopted.

The treatment plan included a preparatory presurgical phase (prophylaxis and oral hygiene instruction), followed by a surgical phase and a final prosthetic phase. The final goal being to reduce the gummy smile by surgically increasing the coronal height and harmonizing the anterior cervical lines and by prosthetically using 8 lithium disilicate veneers and 2 zirconia crowns (on teeth \#15 and 25) to enhance the smile and the teeth shape.

\section{Preparatory Pre-Surgical Phase}

Extra and intra-oral photographs were taken in order to design a digital smile using the Keynote program. Photos were imported to the keynote presentation and a customized digital study was designed for the patient(Fig. 4). A calibrated ruler was used based on the clinical teeth measurements. Future gingival levels adapted to the digitally drawn final clinical crown length were set. Thus the estimated amount of excessive gingiva (and underlying bone) to be removed in crown lengthening based on the final gingival levels, were determined and communicated to the Periodontist.

\section{Surgical Phase}

The surgical procedure was conducted under local anesthesia (2\% lidocaine HCL injection with 1:100,000 epinephrine). Submarginal incisions were made around all teeth from tooth number 15 to 25 and extended one more tooth mesially and distally.

Full thickness flaps were elevated and bone reduction including osteotomy and osteoplasty placing bone levels
$3 \mathrm{~mm}$ from the future teeth margins was performed. Surgical end cutting and round burs of different sizes and types (Surgical carbide contour, diamond contour and diamond finish) were used. Absorbable interrupted 4.0 sutures (Fig. 5) (ACE 4-0 PGA Sutures, ACE Surgical Supply Co, Inc. 1034 Pearl St, Brockton, MA 02301, USA) were used for flap closure with vertical mattress sutures. The patient was asked to take amoxicillin $500 \mathrm{mg}$, three times a day for 10 days or clindamycin 300 mg twice daily for 10 days for penicillin-allergic patients. The patient was also asked to take ibuprofen $400 \mathrm{mg}$ three times a day for two days for adequate pain control and to rinse with $0.2 \%$ chlorhexidine gluconate three times a day for two weeks. Sutures were removed at one week, (Fig. 6) and the patient placed for 4 months, on a strict 3 weeks follow-ups for periodontal maintenance and control (Fig. 7).

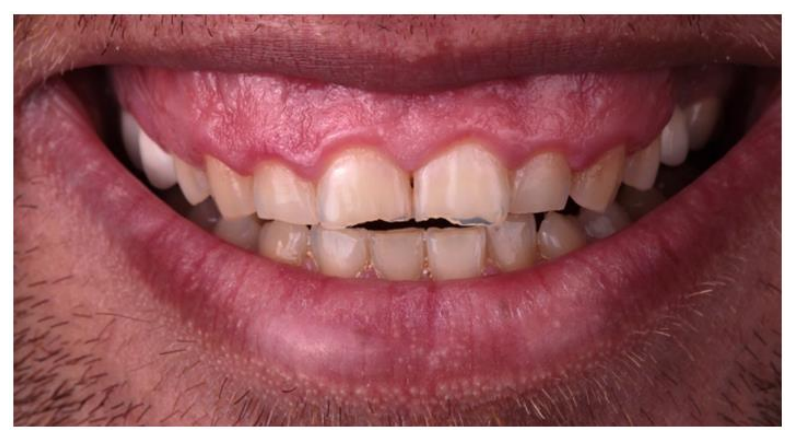

Fig. 1: Initial situation with an excessive gingival display of the patient's smile. Frontal view
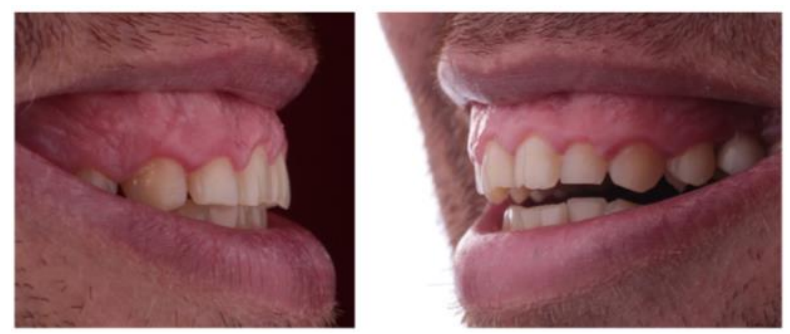

Fig. 2: Initial situation with an excessive gingival display of the patient's smile. Lateral view

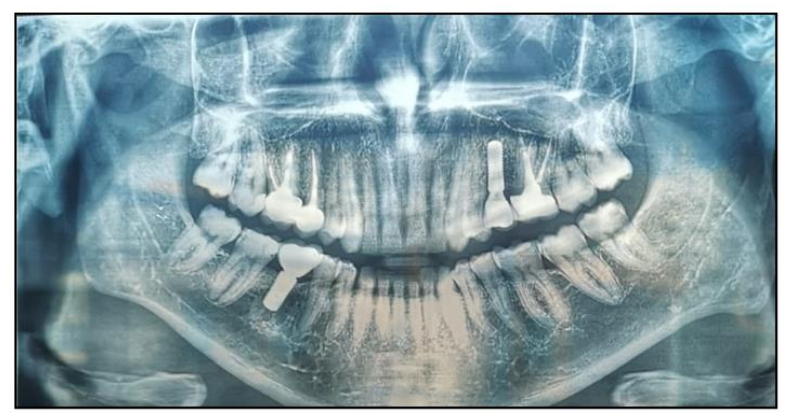

Fig. 3: Panoramic X-ray 


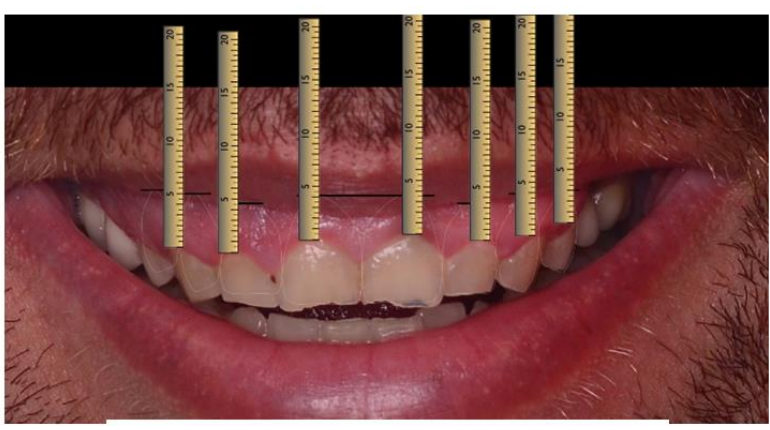

Fig. 4: Future gingival levels placed on a keynote presentation

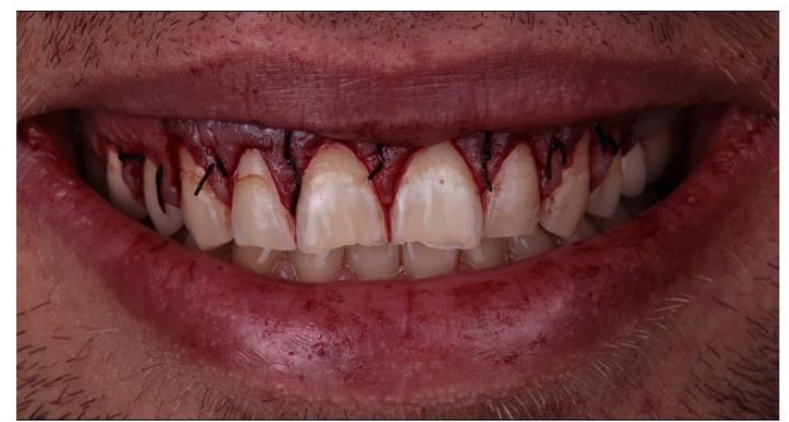

Fig. 5: Clinical situation after esthetic crown lengthening procedure

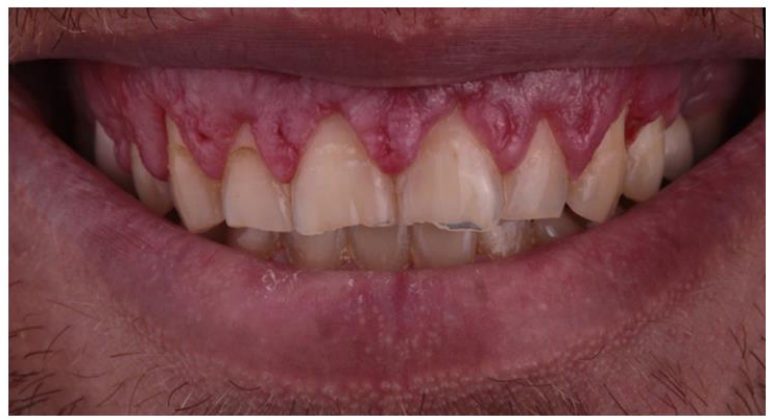

Fig. 6: Ten days post-surgical at sutures removal

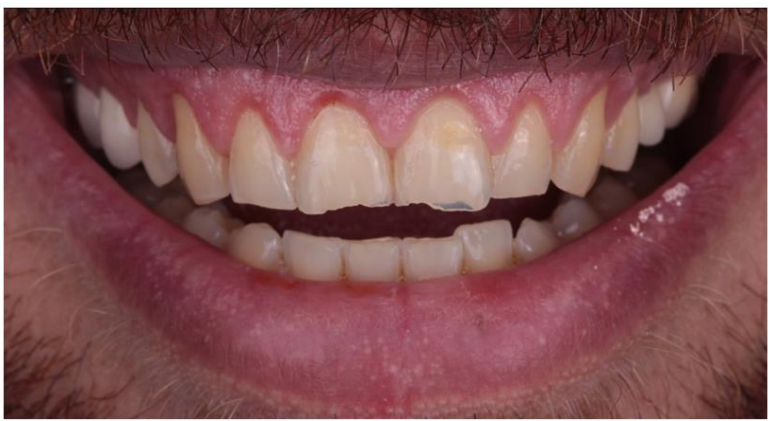

Fig. 7: Four-month post-surgery

\section{Prosthetic Phase}

After a period of 4-month healing, alginate impressions were taken and poured. Casts were mounted on an articulator (Quick® Master) and a wax-up made from the upper right second bicuspid to the upper left second bicuspid. Based on the wax-up, a silicon index was prepared (3M Express STD VPS Impression Putty, Maplewood, Minnesota, U.S). This allowed us to do a mock-up for the patient using a temporary material (Luxatemp star DMG, Hambourg, Germany). This mockup simulated the final restorations, thus allowing the patient to have a preview of the final result and served as a guide for teeth preparation and temporization.

\section{Teeth Preparation}

Once the mock-up in mouth, initial depth grooves were placed on the facial surface, using a gauge bur (\# 834-FG028 ) of $0.5 \mathrm{~mm}$ on the middle and incisal thirds and of 0.3 $\mathrm{mm}$ on the cervical third. These grooves were made from mesial to distal, following three different inclinations (cervical, middle and incisal third) according to the anatomy of the labial surface. On the incisal edge, $1.5 \mathrm{~mm}$ incisal depth cuts were made on the mockup using an axial reduction bur (MADC 015 bur. Kerr west Collins IOA, USA). The bottom of the labial grooves were then colored for contrast with a pencil to ensure a reduction no more than the grooves depth. A chamfer diamond bur was used for the buccal reduction performed following the three inclinations (Cervical, middle and incisal third) until a uniform, homothetic labial surface thickness was reached. Interproximal penetrations (mesial and distal) of $1 \mathrm{~mm}$ depth were done, providing a box-like feature to facilitate the veneer positioning. The contact points of all preparations were broken. The final incisal reduction was done using the diamond bur (\#852 G 806 314). All teeth were prepared in enamel. There was no dentine exposure in all the preparations in order to be conservative as much as possible. Any excess of the provisional mockup material was removed (Fig. 8).

On tooth \# 15, the old crown was removed and the preparation was adjusted. On tooth \# 25 the old crown on implant was removed and a closed-tray impression coping was placed.

\section{Impression}

One step impression technique was applied using a type A polyvinyl siloxane material (DMG, America). Retracting cord (\#0) was gently inserted in the gingival sulcus around the labial and palatal surfaces of the teeth. Final margin preparations were than slightly submarginally positioned and polished while sharp angles were rounded (Fig. 9).

A second retracting cord (\#01) was inserted over the first one to ensure a good gingival retraction, leaving a 
small tag for easy removal. While the first retracting cord (\#0) is left in situ, for mechanical homeostasis, the second retracting cord (\#01) was removed almost simultaneously with a light impression material injection into the sulcus and around the teeth, followed immediately by the heavy impression material loaded in the impression tray that was inserted in the patient mouth and left under stable digital pressure for $3 \mathrm{~min}$, based on the manufacturer's recommendations. A regular alginate impression of the lower arch was also taken. Both impressions were rinsed under running water for debris and exudate removal before being immersed for $20 \mathrm{~min}$ in $2.5 \%$ Glutaraldehyde solution for disinfection, rinsed and dried prior to packaging.

B1 shade was selected using a Vita classical color shade guide and a new temporary was made by adding again provisional material (Luxatemp star DMG, Hamburg, Germany) in the same silicone index (3M Express STD VPS Impression Putty, Maplewood, Minnesota, U.S.) previously used and inserted into the patient mouth to cover the prepared teeth. Excess material was removed before the provisional material set, index removed after material setting and occlusion was adjusted on the new mock-up (Fig. 10).

\section{Try-In}

Before try in, temporaries were removed and teeth surfaces perfectly cleaned from any residual resin cement or provisional material. Veneers were tried individually first, without any try-in material. Buccal, palatal and proximal marginal fits were visually detected using a magnifier and a probe (Figs. 11 and 12).

Once all veneers fitting was checked, a try-in material (Ivoclar Vivadent Esthetic lc, Schaan, Liechtenstein) was used to hold the veneers in place, in order to show the patient and check for his final approval of esthetics (Figs. 13 to 16).

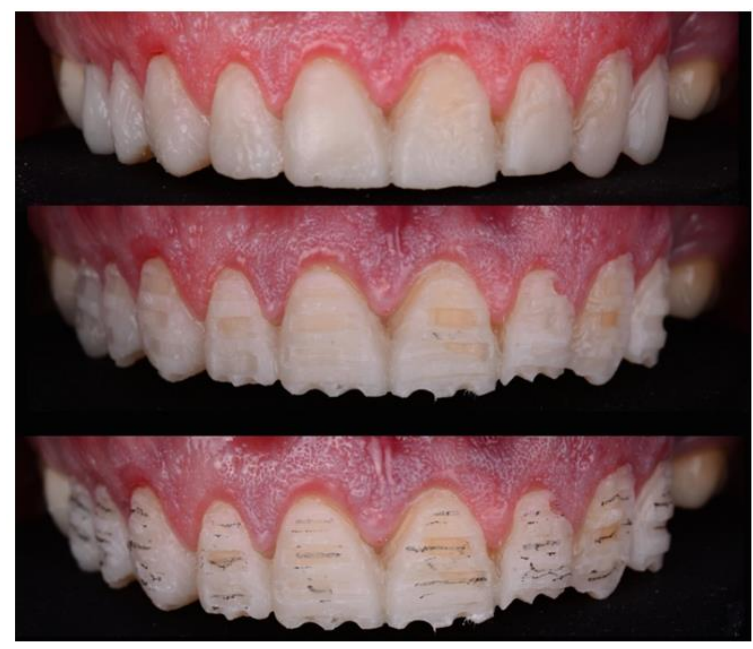

Fig. 8: Mockup with depth gauge preparations

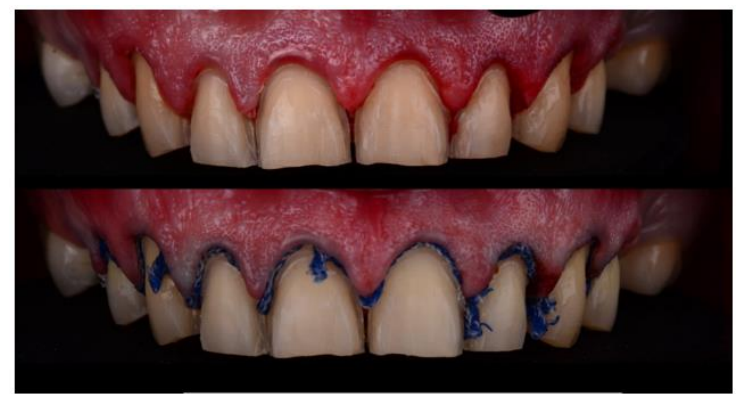

Fig. 9: Prosthetic phase (preparations, retraction cords placement

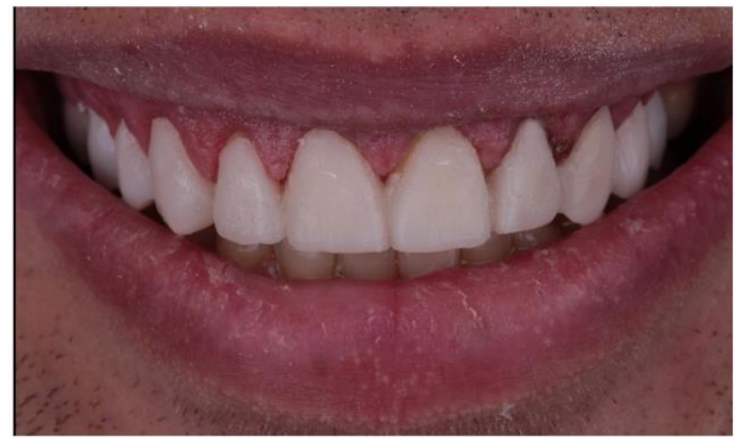

Fig. 10: Temporary teeth placement

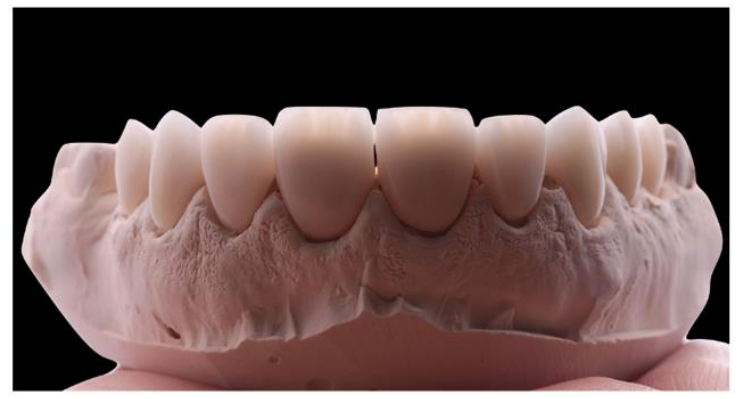

Fig. 11: Ceramic Veneers

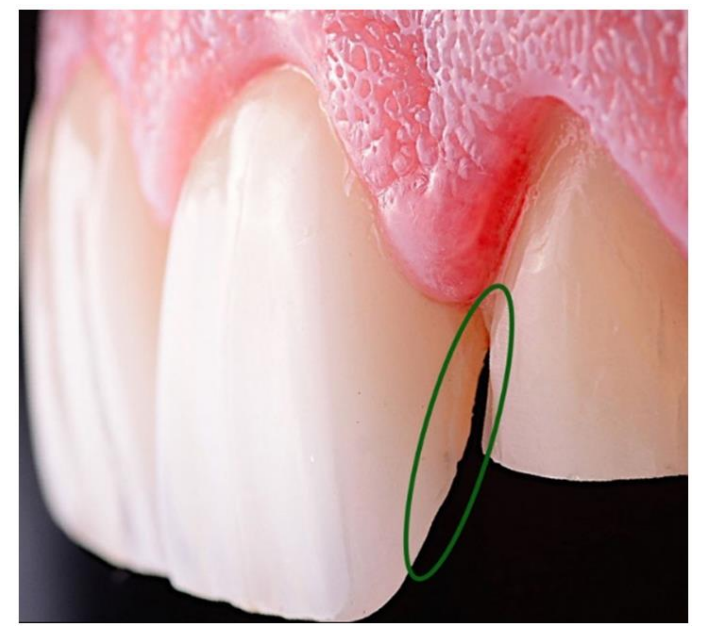

Fig. 12: Veneer's adaptation to the tooth 


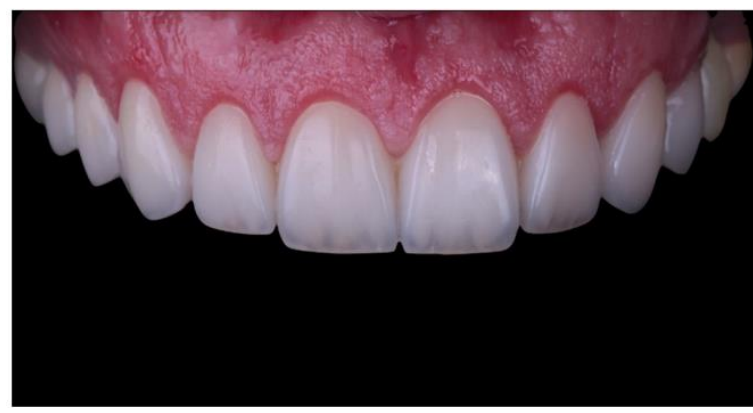

Fig. 13: Post-operative intra oral result

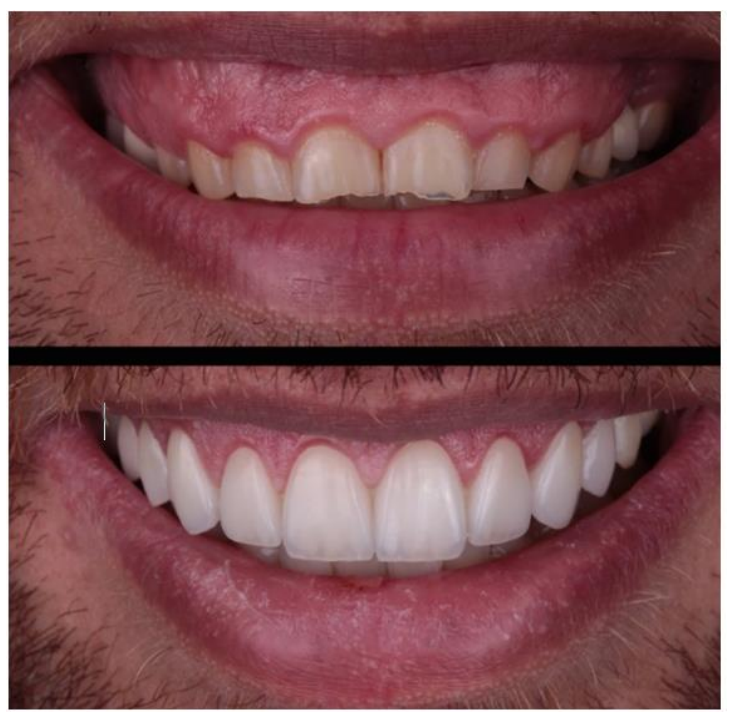

Fig. 14: Pre-operative and post-operative smile. Frontal view

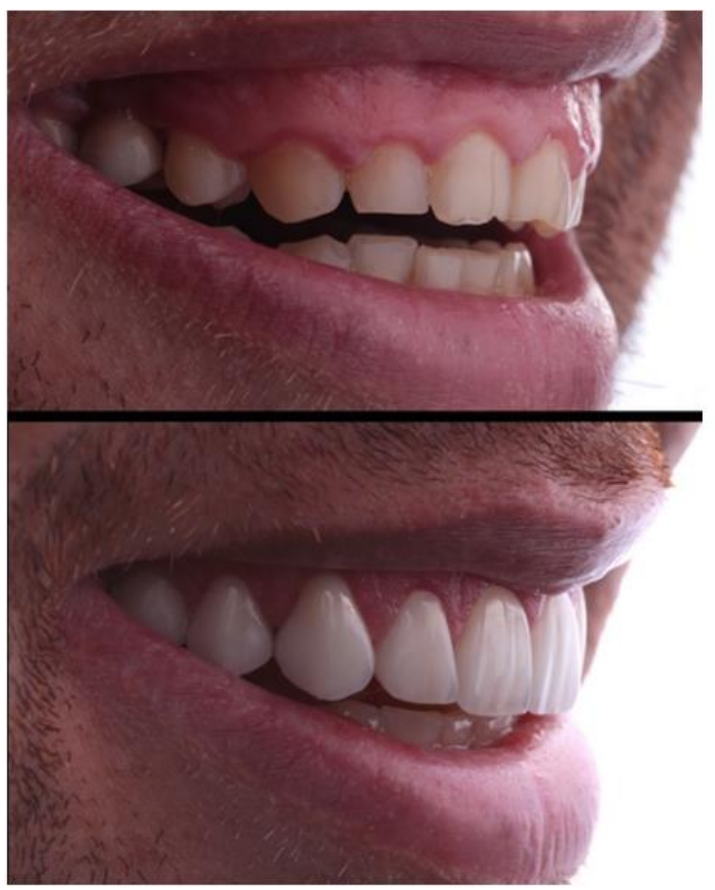

Fig.15: Pre-operative and post-operative smile. Lateral view

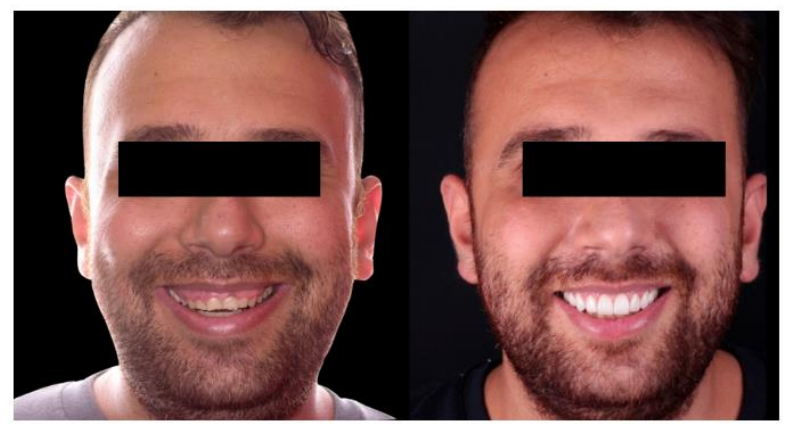

Fig. 16: Pre-operative and post-operative smile. Full face view

\section{Bonding Procedure}

In order to ensure a dry and well retracted field, a specific rubber dam (optraDam from Ivoclar vivadent, Schaan, Liechtenstein) was placed. Each veneer was to be individually bonded in order to secure a perfect adaptation and a perfect cleaning of the excess cement and prior to each veneer bonding, adjacent teeth were covered by a Teflon tape.

\section{Teeth Preparation}

The prepared tooth was etched with $37 \%$ phosphoric acid gel (Total etch, Ivoclar Vivadent, Schaan, Liechtenstein) for $30 \mathrm{sec}$ on enamel. Then the tooth was rinsed for $30 \mathrm{sec}$ and carefully dried. A thin layer of bonding (Adhese universal Ivoclar Vivadent, Schaan, Liechtenstein) was applied, gently air-dried and light polymerized for $20 \mathrm{sec}$.

\section{Veneer Preparation}

The veneer was cleaned with Ivoclean (Ivoclar Vivadent, Schaan, Liechtenstein) as a first step for $20 \mathrm{sec}$, then rinsed and dried. Monobond etch and prime (Ivoclar Vivadent, Schaan, Liechtenstein) was applied on the veneer surface with a microbrush and coated for $20 \mathrm{sec}$. The Monobond etch and prime was left on the veneer surface for another $40 \mathrm{sec}$ then rinsed with water and air-dried (according to the manufacturer 's instructions).

\section{Final Cementation}

Variolink LC, a light-cured resin cement (Ivoclar Vivadent esthetic 1c, Schaan, Liechtenstein) was used for final cementation. The veneer was seated with finger pressure and light cured with light of $1200 \mathrm{~mW} / \mathrm{cm}^{2}$ intensity for $2 \mathrm{sec}$ using the Bluephase style light (Ivoclar Vivadent, Schaan, Liechtenstein). Excess cements were removed with a probe on the buccal and palatal margins, while a floss was used to remove the interproximal excess. Glycerin gel (liquid strip, Ivoclar Vivadent, Schaan, Liechtenstein) was then applied on the cervical part to prevent an oxygen-inhibited layer of composite resin to form and a $20 \mathrm{sec}$ additional 
polymerization was done. Teflon tapes were removed from the adjacent teeth and the contact point with the next veneer was checked. The same procedure was repeated to cement the rest of the veneers. A final polymerization was performed for $40 \mathrm{sec}$ on all surfaces, once the cementation of the eight veneers was done.

The zirconia crown on tooth \#15 was cemented with a resin cement (RelyX ultimate $3 \mathrm{M}$ ESPE) and with a temporary cement (Temp-Bond TM, Kerr) on the implant \#25.

\section{Discussion}

In this case report, we presented a case of a diagnosed Altered Passive Eruption (APE), classified as a type 1 subgroup B, according to (Coslet et al., 1977). The diagnosis was based on clinical and radiographic examinations. The clinical examination included a complete periodontal charting comprising Probing Pocket Depth (PPD), Recession Defects (RD) and thus Clinical Attachment Loss (CAL) with teeth mobility assessment, Full Mouth Plaque Scores (FMPS) and Full Mouth Bleeding Index (FMBI). One of the clear limitations in this case was the lack of additive radiographic examinations including periapical and bitewing radiographs. The patient was adamant and categorically refused to have more X-rays taken to help confirm the Diagnosis and we were limited to the use of the only Panoramic available.

The treatment consisted of three phases: A preoperative, a surgical and a prosthetic phase.

Concerning the pre-operative phase, it included essentially a digital approach to the case. We imported the clinical pictures to a software (Keynote), calibrated the ruler and digitally drew the final restorations with their apical margins. This allowed us to have the exact measures needed to determine the future teeth length (And the gingival margins), thus communicating to the periodontist the needed crown lengthening ().

In this case, the surgical phase consisted only of a crown lengthening. Different treatment options are available when a gummy smile is diagnosed. They include major maxillo-facial surgery (When it is due to a maxillary vertical excess or overgrowth), a crown lengthening, a surgical lip repositioning, Botox or other substitutes injections and full mouth prosthetic reconstruction. In severe cases, it might include a combination of therapies. The success of a gummy smile treatment is thus essentially based on the proper diagnosis, in order to choose the adequate surgical approach when necessary. In fact, while a gummy smile resulting from a short hypertonic lip can be treated with a Botulinum Toxin A injection or a surgical lip repositioning, a gummy smile resulting from an APE can be treated with either a gingivectomy, or a crown lengthening (Wongsirichat, 2019). Obviously some combination therapies are required in some cases and have proven to be successful (Mostafa,2018; Ramesh et al.,2019). In the case of a gummy smile due to an APE, the surgical approach can differ depending on the classification as described by (Coslet et al., 1977; Levine and McGuire, 1997). In our surgical approach consisting of a crown lengthening, care was taken to recreate a scalloped architecture that led to a homogenous scalloped gingival architecture as prescribed by different authors (Pontoriero, 2014; Nowzari, 1998; Mostafa,2018). One could argue that a surgical stent could have been used. In our case, the projected CEJ position was pre-set by the digital smile design used, where a 2-3 mm apical displacement of the margins was needed. This does not preclude the fact that using a stent could be advantageous (De Oliveira et al., 2015).

Concerning the prosthetic part, since only veneers were planned, no teeth preparation and temporary crowns were done during the surgery. In this case, teeth preparation was done and impressions taken 4 months after crown lengthening in accordance with the positive clinical exam done by the surgeon where complete clinical healing of the periodontium was observed. In surgically involved cases, the timing of prosthetic therapy initiation is essential and related to the final positioning of the gingival margin (Hempton and Dominici, 2010). Several authors advocate a healing period ranging between 1 to 3 months or even up to 6 months in the anterior zone (Ardakani et al., 2016; Vaziriet al., 2015; Arora et al., 2013).In fact there are no specific guidelines, but only general guidelines depending on the area where the crown lengthening was performed (anterior or posterior), the amount of bone removed, the biotype of the periodontium, the post suturing flap position and general oral hygiene of the patient (Vaziri et al., 2015). In general, as shown by 6 months after crown lengthening, periodontal tissues are considered stable, with minimal or no significant changes in the level of the gingival margin margin (Lanning et al., 2003; Brägger et al., 1992).

When preparing teeth for Veneers, options for the preparation of the interproximal area range from no preparation to a preparation that stops just before the contact area to a slight opening of the interproximal contact, to a complete removal of the contact point (Vanlıoglu and Kulak-Özkan, 2014), but there are no conclusive evidence or guidelines that can be found concerning the best way of the interproximal area preparation.

Removing contact points of all preparations, in the most conservative way, as it was done in this case, has many advantages, it allows freedom to the laboratory to well adjust the contours and position of ceramic veneers, increases surface area of bonding, reduces the risk of visible interproximal staining at margins and hides the junction tooth/veneer (Vanlıoğlu and Kulak-Özkan, 2014). We placed the gingival margins slightly sub gingivally to hide the teeth color and care was taken to remove excess cement before final setting to avoid any subsequent 
inflammation. According to (Costa et al., 2018) when placing the margins slightly sub gingivally in the anterior zones, no difference is found regarding healthy gingival contours when compared to juxta or supragingival margins. Conventionally, the preparation of lithium disilicate ceramic for bonding is done by conditioning with fluoridric acid to promote and increase the ceramic roughness, followed by silane application to promote a durable adhesion (Lise et al., 2015). Fluoridric acid may weaken the ceramic, but it remains the only available technique to condition Lithium disilicate. To this date there is no consensus on the ideal fluoridric acid concentration and etching time needed to avoid its toxicity on ceramics, but manufacturers recommend etching with 5\% HF for $20 \mathrm{sec}$ although these numbers vary across the published literature (Bona et al., 2002; Kalavacharla et al., 2015).

In our case we used the self-etching ceramic primer: Monobond etch and prime (Ivoclar Vivadent). According to the manufacturer, this product reduces the toxic effect of fluoridric acid on ceramic, reduces the chair-time required and the technique sensitivity of etching ceramic when compared to the conventional methods (Blatz et al., 2018; Baader et al., 2016).

Numerous articles compared the immediate bond strength values when using the self-etching ceramic primer and the twostep fluoridric acid and silane. They showed similar immediate bond strength values. Nevertheless, further studies are needed in order to assess and guarantee the long-term bond strength of the monobond etch and prime when compared to the conventional technique (Siqueira et al., 2016; El-Damanhoury et al., 2018).

\section{Conclusion}

A smile is the most common facial expression used to display happiness and satisfaction (Tulika et al., 2017). We reported a case of a gummy smile related to a Type 1 subgroup B that was pre-treatment planned digitally and treated with a combination of crown lengthening and veneers, with esthetically pleasing results.

\section{Author's Contributions}

Farah Antar: Performed the case and helped in writing the manuscript.

Rasha Hajj and Maroun Dagher: Wrote the manuscript. Elie Zebouni: Reviewed the manuscript

\section{Ethics}

This article is original and contains unpublished material. The corresponding author confirms that all of the other authors have read and approved the manuscript and no ethical issues involved.

\section{References}

Allen, E. P. (1988). Use of mucogingival surgical procedures to enhance esthetics. Dental Clinics of North America, 32(2), 307-330. https://europepmc.org/article/med/3163980

Alpiste-Illueca, F. M. (2011). Altered passive eruption (APE): A little-known clinical situation. https://doi.org/10.4317/medoral.16.e100

Ardakani, M. R. T., Khalilian, F., Nateghi, Z., Esmaeilnejad, A., \& Janbakhsh, N. (2016). A review of the crown lengthening surgery; the basic concepts. Journal of Advances in Medicine and Medical Research, 1-7. https://doi.org/10.9734/BJMMR/2016/22602

Arora, R., Narula, S. C., Sharma, R. K., \& Tewari, S. (2013). Evaluation of supracrestal gingival tissue after surgical crown lengthening: a 6-month clinical study. Journal of Periodontology, 84(7), 934-940. https://doi.org/10.1902/jop.2012.120162

Baader, K., Hiller, K. A., Buchalla, W., Schmalz, G., \& Federlin, M. (2016). Self-adhesive luting of partial ceramic crowns: Selective enamel etching leads to higher survival after 6.5 Years in Vivo. Journal Adhes Dental, 18(1), 69-79. http://www.quintpub.com/userhome/jad/jad_18_1_b aader_p69.pdf

Blatz, M. B., Vonderheide, M., \& Conejo, J. (2018). The effect of resin bonding on long-term success of high-strength ceramics. Journal of Dental Research, 97(2),

132-139. https://doi.org/10.1177/0022034517729134

Bona, A. D., Anusavice, K. J., \& Hood, J. A. (2002). Effect of ceramic surface treatment on tensile bond strength to a resin cement. International Journal of Prosthodontics, 15(3).

Brägger, U., Lauchenauer, D., \& Lang, N. P. (1992). Surgical lengthening of the clinical crown. Journal of Clinical Periodontology, 19(1), 58-63. https://doi.org/10.1111/j.1600-051X.1992.tb01150.x

Coslet, J. G., Vanarsdall, R., \& Weisgold, A. (1977). Diagnosis and classification of delayed passive eruption of the dentogingival junction in the adult. The Alpha Omegan, 70(3), 24-28. https://europepmc.org/article/med/276255

Costa, V. L. S., Tribst, J. P. M., Uemura, E. S., de Morais, D. C., \& Borges, A. L. S. (2018). Influence of thickness and incisal extension of indirect veneers on the biomechanical behavior of maxillary canine teeth. Restorative Dentistry \& Endodontics, 43(4). https://doi.org/10.5395/rde.2018.43.e48

de Oliveira, P. S., Chiarelli, F., Rodrigues, J. A., Shibli, J. A., Zizzari, V. L., Piattelli, A., ... \& Perrotti, V. (2015). Aesthetic surgical crown lengthening procedure. Case Reports in Dentistry, 2015. https://doi.org/10.1155/2015/437412 
El-Damanhoury, H. M., \& Gaintantzopoulou, M. D. (2018). Self-etching ceramic primer versus hydrofluoric acid etching: Etching efficacy and bonding performance. Journal of Prosthodontic Research, $62(1)$ $75-83$. https://doi.org/10.1016/j.jpor.2017.06.002

Garber, D. A., \& Salama, M. A. (1996). The aesthetic smile: diagnosis and treatment. Periodontology 2000, 11(1), 18-28. https://doi.org/10.1111/j.16000757.1996.tb00179.x

Hempton, T. J., \& Dominici, J. T. (2010). Contemporary crown-lengthening therapy: a review. The Journal of the American Dental Association, 141(6), 647-655. https://doi.org/10.14219/jada.archive.2010.0252

Kalavacharla, V. K., Lawson, N. C., Ramp, L. C., \& Burgess, J. O. (2015). Influence of etching protocol and silane treatment with a universal adhesive on lithium disilicate bond strength. Operative Dentistry, 40(4), 372-378. https://doi.org/10.2341/14-116-L

Lanning, S. K., Waldrop, T. C., Gunsolley, J. C., \& Maynard, J. G. (2003). Surgical crown lengthening: evaluation of the biological width. Journal of Periodontology, 74(4), 468-474 https://doi.org/10.1902/jop.2003.74.4.468

Levine, R. A., \& McGuire, M. (1997). The diagnosis and treatment of the gummy smile. Compendium of Continuing Education in Dentistry (Jamesburg, NJ: 1995), 18(8), 757-762. https://europepmc.org/article/med/9533335

Lise, D. P., Perdigão, J., Van Ende, A., Zidan, O., \& Lopes, G. C. (2015). Microshear bond strength of resin cements to lithium disilicate substrates as a function of surface preparation. Operative Dentistry, 40(5), 524-532. https://doi.org/10.2341/14-240-L

Madaan, V., Kini, V. V., Yadav, S. R., \& Padhye, A. M. (2015). Altered passive eruption: Report on management of two cases. JournalContempt Dentistry, 5(3), 173-177. https://doi.org/10.5005/jpjournals-10031-1130

Mostafa, D. (2018). A successful management of sever gummy smile using gingivectomy and botulinum toxin injection: A case report. International Journal of Surgery Case Reports, 42, 169-174. https://doi.org/10.1016/j.ijscr.2017.11.055
Nowzari, H. (1998). Esthetic periodontal therapy. Compendium of Continuing Education in Dentistry (Jamesburg, NJ: 1995), 19(5), 463-468. https://europepmc.org/article/med/9693509

Pontoriero, R. (2014). Osseous surgery in periodontal treatment. The International Journal of Esthetic Dentistry, 9(2), 268-277. https://pubmed.ncbi.nlm.nih.gov/24765633/

Ramesh, A., Vellayappan, R., Ravi, S., \& Gurumoorthy, K. (2019). Esthetic lip repositioning: A cosmetic approach for correction of gummy smile-A case series. Journal of Indian Society of Periodontology, 23(3), 290. https://doi.org/10.4103/jisp.jisp_548_18

Siqueira, F. S., Alessi, R. S., Cardenas, A. F., Kose, C., SC, S. P., Bandeca, M. C., ... \& Gomes, J. C. (2016). New Single-bottle Ceramic Primer: 6-month Case Report and Laboratory Performance. The Journal of Contemporary Dental Practice, 17(12), 1033-1039.

https://doi.org/10.1016/j.prosdent.2018.12.018

Tulika, S., Bhongade, M.L., Supria, G., Bhairavi, K., \& Ankita, A. (2017). Management of altered passive eruption: Review \& case report. Scholars Journal of Dental Science, 4(3), 104-108 http://saspjournals.com/wpcontent/uploads/2017/04/SJDS-43104-108.pdf

Vanlığlu, B. A., \& Kulak-Özkan, Y. (2014). Minimally invasive veneers: current state of the art. Clinical, Cosmetic and Investigational Dentistry, 6, 101. https://doi.org/10.2147/CCIDE.S53209

Vaziri, F., Haerian, A., Lotfi Kamran, M. H., \& Abrishami, M. (2015). Evaluation of the effect of surgical crown lengthening on periodontal parameters. Journal of Dental Materials and Techniques, 4(3), 143-148. https://jdmt.mums.ac.ir/article_4597_0.html

Wongsirichat, N. (2019). Gummy smile: a review of etiology, manifestations and treatment. Sirijaj Medical Journal, 71(2), 168-174. https://doi.org/10.33192/Smj.2019.26 